\title{
Gemeinsame Entwicklung von Leichtbau-Technologien
}

BMW und die Toyota Motor Corporation (TMC) haben Ende Januar 2013 einen „Vertrag zur gemeinsamen Entwicklung von Brennstoffzelle, Architektur und Komponenten eines Sportwagens sowie zur gemeinsamen Forschung und Entwicklung von Leichtbau-Technologien" unterzeichnet. Somit setzen die Partner ihre strategische Zusammenarbeit im Bereich der nachhaltigen Mobilität fort. Diese Vereinbarung folgte auf die bereits im Juni vergangenen Jahres unterzeichnete Absichtserklärung. Die Automobilhersteller wollen gemeinsam Leichtbau-Technologien für den Karosseriebau unter Verwendung neuester Materialien entwickeln. Diese Techniken sollen bei der gemeinsamen Entwicklung einer Sportwagen-Plattform sowie in anderen Fahrzeugen von BMW und TMC zum Einsatz kommen. Zudem haben sich die Partner darauf verständigt, bis Ende 2013 eine Machbarkeitsstudie zu einer gemeinsamen Plattform für einen mittelgroßen Sportwagen durchzuführen. Die Unternehmen streben an, ihre jeweiligen Technologien und Fertigkeiten auf hohem Niveau abzustimmen, um den Kunden die bestmögliche Lösung anbieten zu können, heißt es in einer gemeinsamen Mitteilung. Beide Unternehmen beabsichtigen, im Bereich der Sportwagen-Entwicklung weiterhin zusammenzuarbeiten.
Zudem ist aus Sicht der beiden Unternehmen die Brennstoffzellen-Technik eine wesentliche Voraussetzung, um Null Emissionen zu erreichen. BMW und TMC wollen daher gemeinsam ein grundlegendes Brennstoffzellen-Fahrzeugsystem entwickeln, das auch die Bereiche Wasserstofftank, Motor und Batterie umfasst. Die Entwicklung soll 2020 abgeschlossen sein. Außerdem wollen beide Unternehmen für die Entwicklung der Wasserstoff-Infrastruktur gemeinsame Codes und Standards entwickeln, die für die weitere Verbreitung von Fahrzeugen mit Brennstoffzellen-Technik erforderlich sind.

\section{$85 \%$ SCHNELLER \\ NEUE ZENTRIFUGENTECHNIK ERMÖGLICHT PRÜFUNG VON KLEB-, HAFT- UND VERBUNDFESTIGKEITEN}

Klebeverbindungen, Beschichtungen und Kompositwerkstoffe lassen sich nun mit einer neuartigen analytischen Zentrifuge untersuchen. Die Idee der Wissenschaftler an der BAM Bundesanstalt für Materialforschung und -prüfung, die sich hinter der neuen Technik verbirgt, ist, dass die Proben nicht mehr in die Prüftechnik eingespannt sondern nur noch eingesteckt werden müssen. Durch das einfache Einstecken der Prüfkörper sei eine sehr schnelle Beladung möglich. Zudem sollen sich bis zu acht Proben gleichzeitig unter identischen Bedingungen prüfen lassen.

Herkömmliche Zugprüfmaschinen waren und sind nur in der Lage, einen (eingespannten) Probekörper zu testen. Das von BAM-Forschern bereits 2004 zum Patent angemeldete Funktionsprinzip wurde seitdem von dem Berliner Unternehmen LUM als Lizenznehmer zum marktreifen Produkt weitereinwickelt.
Die resultierende Zeitersparnis soll im Ergebnis bei etwa $85 \%$ liegen. Wenn die Prüfstempel abreißen, beziehungsweise der Verbund versagt, werden der Zeitpunkt, die aktuelle Rotationsgeschwindigkeit und der betroffene Probenplatz automatisch aus der laufenden Zentrifuge an einen Computer gesendet. Eine speziell entwickelte Software berechnet online die kritischen Kräfte und Zugspannungen und stellt die Ergebnisse dar.

Das neue Prüfsystem sieht auf den ersten Blick wie eine herkömmliche Tischzentrifuge aus. In ihr läuft ein von LUM entwickelter Spezialrotor mit bis zu 13.000/min. Durch aufgeklebte oder anderweitig mit dem Prüfkörper verbundene Prüfstempel können Klebungen, Beschichtungen und Kompositwerkstoffe mit Zugkräften belastet werden. Wie bei einem Karussell wird die aus der Drehbewegung resultierende Zentrifu- galbeschleunigung ausgenutzt. Es werden Beschleunigungen von bis zu $10.000 \mathrm{~g}$ erreicht, dabei wirken auf die Proben Fliehkräfte von bis zu 6,5 kN.

„Durch die durchgehend realisierbaren Prüfkräfte von 0,1 N bis 6,5 kN sind nahezu beliebige Prüfabläufe sowohl für Kurzzeitbeanspruchungs- als auch Langzeitermüdungstests programmierbar", erklärt Professor Dietmar Lerche, Geschäftsführer des auf analytische Zentrifugen spezialisierten Unternehmens LUM in Berlin. BAM-Fachbereichsleiter Uwe Beck ergänzt: „Das vollautomatisierte AufTisch-Prüfsystem ist sowohl für Forschung und Entwicklung als auch für die industrielle Qualitätssicherung gleichermaßen interessant". Der Durchmesser der Prüffläche kann bis zu $10 \mathrm{~mm}$ betragen, die maximalen Probenabmessungen liegen derzeit bei $30 \mathrm{~mm} \times 30 \mathrm{~mm} \times 15 \mathrm{~mm}$. Die dabei prüfbare Zugfestigkeit beträgt bis zu 82,5 $\mathrm{N} / \mathrm{mm}^{2}$. 